\title{
Improvement of notch fatigue properties of ultra-high CM400 maraging steel through shot peening - CORRIGENDUM
}

\author{
Qi-qiang Duan, Bin Wang, Peng Zhang, Ke Yang, and Zhe-Feng Zhang \\ doi: https://doi.org/10.1557/jmr.2017.358, Published by Cambridge University Press, 11 September \\ 2017
}

In Duan et al. ${ }^{1}$, additional affiliation information for Qi-qiang Duan was inadvertently omitted. The affiliations for Qi-qiang Duan are Shenyang National Laboratory for Materials Science, Institute of Metal Research, Chinese Academy of Sciences, 72 Wenhua Road, Shenyang 110016, China, and University of Chinese Academy of Sciences, 19 Yuquan Road, Beijing, 100049, China. The authors regret this error.

\section{REFERENCE}

1. Q. Duan, B. Wang, P. Zhang, K. Yang, and Z.-F. Zhang. Improvement of notch fatigue properties of ultra-high CM400 maraging steel through shot peening. J Mater. Res. (2017). https://doi.org/10.1557/jmr.2017.358. 